\title{
Residual Stress Evaluation of Short-Fiber Reinforced Plastics by X-Ray Diffraction
}

\author{
Keisuke Tanaka ${ }^{1, a^{*}}$, Yuuki Koike ${ }^{1, b}$ and Yoshiaki Akiniwa ${ }^{2, c}$ \\ ${ }^{1}$ Department of Mechanical Engineering, Meijo University, Nagoya, Japan \\ 2Department of Mechanical Engineering, Yokohama National University, Yokohama, Japan \\ aktanaka@meijo-u.ac.jp, bkoi.yu051@gmail.com, cakiniwa@ynu.ac.jp
}

Keywords: Residual stress, X-ray diffraction, Short-fiber reinforced plastics, Micromechanics

\begin{abstract}
The X-ray diffraction method is used to evaluate the residual stress in injection-molded plates of short-fiber reinforced plastics (SFRP) made of crystalline thermoplastics, polyphenylene sulphide (PPS), reinforced by carbon fibers with 30 mass\%. The stress in the matrix in the skin layer was determined using $\mathrm{Cr}-\mathrm{K} \alpha$ radiation with the $\sin ^{2} \psi$ method. The $\mathrm{X}$-ray evaluation of stress in carbon fibers was not possible because of high texture. A new method was proposed to evaluate the macrostress in SFRP from the measurement of the matrix stress. According to micromechanics analysis of SFRP, the matrix stresses in the fiber direction and perpendicular to the fiber direction, and shear stress can be expressed as linear functions of the applied (macro-) stresses in the fiber direction and perpendicular to the fiber direction, and shear stress. The proportional constants are named stress-partitioning coefficients. Using skin-layer strips cut parallel, perpendicular and $45^{\circ}$ to the molding direction, the stress in the matrix was evaluated under the uniaxial applied stress and the stress-partitioning coefficients of the above equations were determined. Once the relations between the macrostress and matrix stress are established, the macrostress in SFRP can be evaluated from the measurements of the matrix stresses using X-rays.
\end{abstract}

\section{Introduction}

Short-fiber reinforced plastics (SFRP) are expected to be used more widely in order to reduce the weight of ground vehicles, because the injection molding process makes high-rate and economical production possible. Their application in fatigue-sensitive components is steadily increasing [1,2]. Since the residual stress might have a big influence on the fatigue strength, the nondestructive evaluation of residual stresses existing in SFRP structures is significant to grantee the structural integrity against fracture and fatigue. X-ray diffraction techniques can evaluate the stress in crystalline materials, and will be applied to SFRP when the matrix or fiber is made of crystalline materials. Polyphenylene sulphide (PPS) is a versatile temperature and chemical resistant, inherently flame retardant crystalline thermoplastics [3]. PPS is usually reinforced by short carbon fibers or glass fibers, and the injection molding process is used in producing parts of ground vehicles.

In the present paper, the X-ray diffraction method is used to evaluate the stress in injection-molded SFRP made of PPS reinforced with carbon fibers. Based on the orientation of carbon fibers, injection molded plates (IMP) can be modeled as three-layered lamella where the core layer is sandwiched by skin layers. The stress in the matrix in the skin layer was evalauted from back diffractions of (200), (111) of PPS using Cr-K $\alpha$ radiation with $\sin ^{2} \psi$ method. A new method is proposed to evaluate the residual stress of SFRP from the matrix stress determined by X-rays.

\section{X-Ray Evaluation of Stress in SFRP}

X-Ray Stress Evaluation of Composites. The $\sin ^{2} \psi$ method is commonly used to determine the surface stress in single-phase polycrystalline materials which have random orientation under the condition of plane stress. The stress can be determined from the slope of the linear relation of diffraction angle $2 \theta$ and $\sin ^{2} \psi$ by multiplying the stress constant $K$ as follows [4]: 


$$
\sigma_{x}=K \cdot M, \quad M=\partial(2 \theta) / \partial\left(\sin ^{2} \psi\right) .
$$

For multiphase materials, the X-ray method can detect the mean stress in each phase, and the macrostress is determined from the phase stresses obtained for all constituent phases using a rule of mixture. For two-phase materials like SFRP, the macrostress, $\sigma^{0}$, is determined from the matrix stress $\langle\sigma\rangle^{m}$ and the fiber stress $\langle\sigma\rangle^{f}$ as

$$
\sigma^{0}=\langle\sigma\rangle^{m}(1-f)+\langle\sigma\rangle^{f} f,
$$

where $f$ is the volume fraction of fibers [4]. To determine the macrostress using above equation, the phase stress in both phases need to be known. The X-ray method of stress evaluation is difficult to apply to amorphous glass fibers or highly textured carbon fibers. Therefore, we propose a new method to determine the macrostress only from the matrix stress.

Macrostress Evaluation of SFRP from Matrix Stress. By modeling fibers in SFRP as prolate spheroids, the stresses in matrix and fiber phases can be calculated as a function of the applied stress based on micromechanics as described in Appendix. Assuming the plane stress state, the matrix stresses parallel and perpendicular to fibers, $\sigma_{1}^{m}$ and $\sigma_{2}^{m}$, and the matrix shear stress, $\tau_{12}^{m}$, are related to the applied (macro-) stresses parallel and perpendicular to fibers, $\sigma_{1}^{A}$ and $\sigma_{2}^{A}$, and the matrix shear stress, $\tau_{12}^{A}$, as follows:

$$
\begin{aligned}
& \sigma_{1}^{m}=\alpha_{11} \sigma_{1}^{A}+\alpha_{12} \sigma_{2}^{A}, \\
& \sigma_{2}^{m}=\alpha_{21} \sigma_{1}^{A}+\alpha_{22} \sigma_{2}^{A}, \\
& \tau_{12}^{m}=\alpha_{55} \tau_{12}^{A},
\end{aligned}
$$

where coefficients $\alpha_{i j}$ indicate the matrix stress partitioned from the applied stress, and can be named stress-partitioning coefficients. In the present paper, these coefficients are determined experimentally, and afterward they will be used to evaluate the macrostress from the matrix stress determined by X-rays.

\section{Experimental Procedure}

Materials and Specimens. The experimental material was SFRP made of PPS reinforced with 30 mass $\%$ of carbon fibers. The volume fraction determined from density was 0.22 . Test specimens were machined from injection-molded plate (IMP) with the thickness of $1 \mathrm{~mm}$. IMP can be regarded as three-layer lamella with the core layer of $0.15 \mathrm{~mm}$ sandwiched by skin layers [5]. The fiber direction of the skin layer is parallel to the molding direction and that of the core layer perpendicular to it. The skin layer plate (SLP) with the thickness of $0.4 \mathrm{~mm}$ was produced by machining IMP from one side only. Strip specimens with $10 \mathrm{~mm}$ width and $55 \mathrm{~mm}$ length were cut out from SLP with the longitudinal directions parallel, perpendicular, and $45^{\circ}$ to the molding direction, and they are called MD (molding direction), TD (transverse direction), and $45^{\circ}$ specimens, respectively.

$\mathrm{X}$-Ray Conditions. The characteristic X-rays of $\mathrm{Cr}-\mathrm{K} \alpha$ radiation was used for stress evaluation. The $\mathrm{X}$-ray equipment used was a high power X-ray generator and parallel beam optics. The only peak with a strong intensity found between $20^{\circ}$ and $160^{\circ}$ was the doublet of (200) and (111) of PPS at $2 \theta=30.53^{\circ}[6,7]$. We use this peak for stress determination, although the sensitivity is because of low diffraction angle. The $\sin ^{2} \psi$ method using the side-inclination arrangement was used for stress determination. The linear absorption coefficients of PPS and SFRP determined experimentally were $125.24 \mathrm{~cm}^{-1}$ and $100.18 \mathrm{~cm}^{-1}$, respectively [5]. The X-ray penetration depth was at most $15 \mu \mathrm{m}$ from the surface.

Determination of Stress-Partitioning Coefficients of SRPR. The load was applied stepwise to three types of SFRP specimens, MD, TD and $45^{\circ}$, in the longitudinal direction. The matrix stress was measured in the longitudinal and transverse directions of each specimen as shown in Fig. 1, where the suffixes 1 and 2 indicate the stresses in the fiber direction and the perpendicular directions, and the 
suffix $\pm 45^{\circ}$ indicates the angle $\pm 45^{\circ}$ to the fiber direction. For MD specimen, the applied stress is $\sigma_{1}^{A}$ and coefficients $\alpha_{11}^{m}, \alpha_{21}^{m}$ can be determined from the matrix stresses, $\sigma_{1}^{m}, \sigma_{2}^{m}$, as a function of the applied stress. Similarly, coefficients $\alpha_{21}^{m}, \alpha_{22}^{m}$ can be determined from the relation between the matrix stress and the applied stress obtained for TD specimen. For $45^{\circ}$ specimen, the applied stress $\sigma_{45}^{A}$ induces the shear stress $\tau_{12}^{A}$. The shear stress in the matrix, $\tau_{12}^{m}$, is determined from the measured values of $\sigma_{+45}^{m}, \sigma_{-45}^{m}$ in the matrix as

$$
\tau_{12}^{m}=\left(\sigma_{+45}^{m}-\sigma_{-45}^{m}\right) / 2 .
$$

From the above two equations, the coefficient of $\alpha_{55}$ is determined by

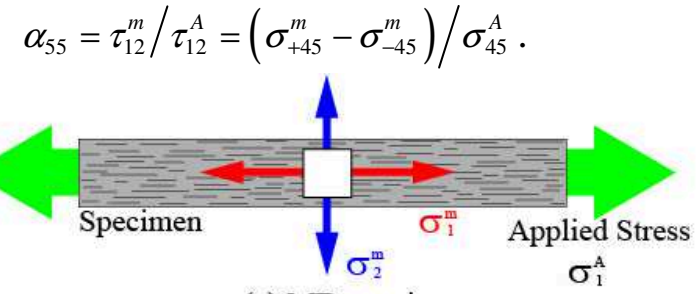

(a) MD specimen

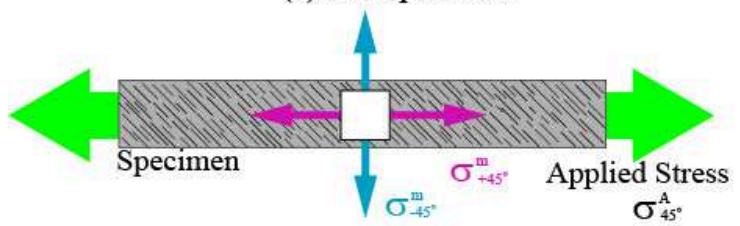

(c) $45^{\circ}$ specimen

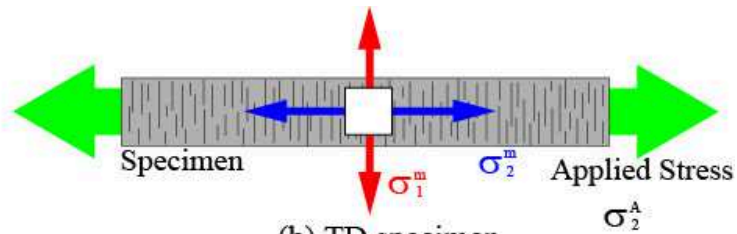

(b) TD specimen

\section{Experimental Results and Discussion}

Stress Measurement of PPS. Fig. 2 shows the $2 \theta-\sin ^{2} \psi$ diagrams obtained from PPS specimens at various applied stresses. Linear regression lines give good approximation to the data for each stress. The slope of $\sin ^{2} \psi$ diagrams changes linearly with the applied stress as shown in Fig. 3, where the bars attached to each data points indicate $68.3 \%$ confidence limit of the linear regression. The stress constant was determined from the change of the slope of $\sin ^{2} \psi$ diagrams with the applied stress as $K=-104.7 \mathrm{MPa} / \mathrm{deg}$.

Matrix Stress of SFRP under Applied Stress. The matrix stress was determined in two directions of $\mathrm{MD}$, TD and $45^{\circ}$ specimens under the applied stress. All data show good linear relations in $2 \theta-\sin ^{2} \psi$ diagrams, and the matrix stress was calculated from the slope of linear regression lines multiplied by the stress constant. Fig. 4 shows the relation between the matrix stress and the applied stress obtained in two directions for MD, TD and $45^{\circ}$ specimens. The bars attached to each data point indicate $68.3 \%$ confidence limit of the linear regression. It is very interesting to notice that the matrix stress at unloaded state is close to zero. Thermal microstress of the matrix due to the mismatch in the coefficient of thermal expansion coefficient between matrix and fiber is negligible in the skin layer, because the penetration depth of X-rays is shallow and the stress relieved near the free surface. This is very important for X-ray stress measurement, otherwise the thermal microstress should be subtracted from the measured matrix stress to evaluate the macrostress of SFRP. The stress components perpendicular to the surface are also negligible for SFRP. Under loading, the matrix stress changes linearly with the applied stress for all cases. From the slope of linear regression, the following relations were obtained, and the stress-partitioning coefficients are determined as follows:

$$
\begin{gathered}
\sigma_{1}^{m}=0.2035 \sigma_{1}^{A}+0.3212 \sigma_{2}^{A}, \\
\sigma_{2}^{m}=0.0111 \sigma_{1}^{A}+0.8595 \sigma_{2}^{A}, \\
\tau_{12}^{m}=0.2761 \tau_{12}^{A} .
\end{gathered}
$$




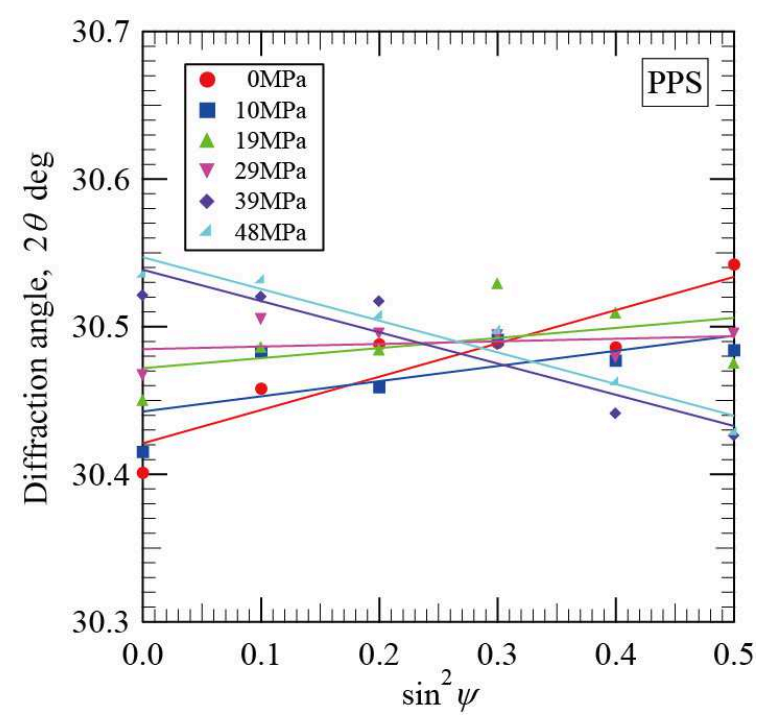

Fig. $22 \theta-\sin ^{2} \psi$ diagram of PPS under applied stress.

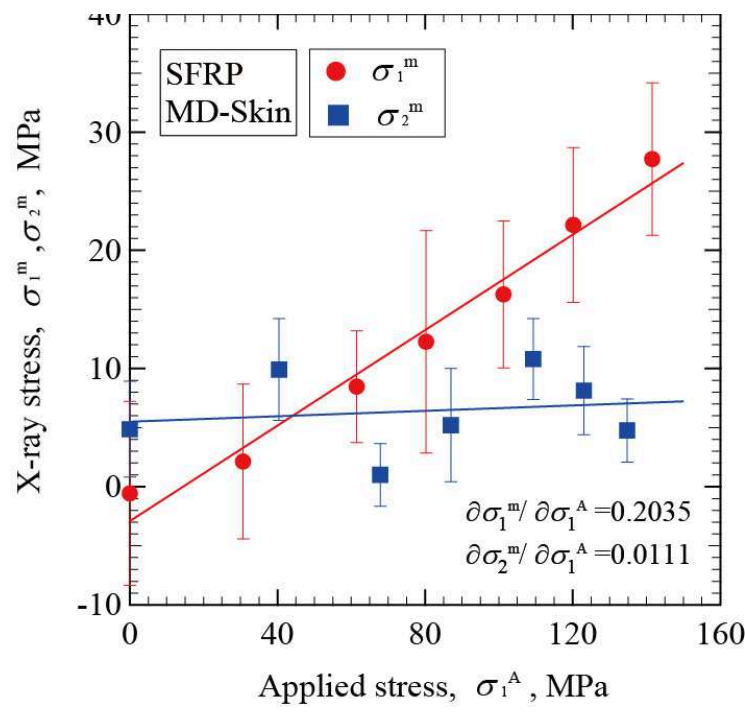

(a) MD specimen

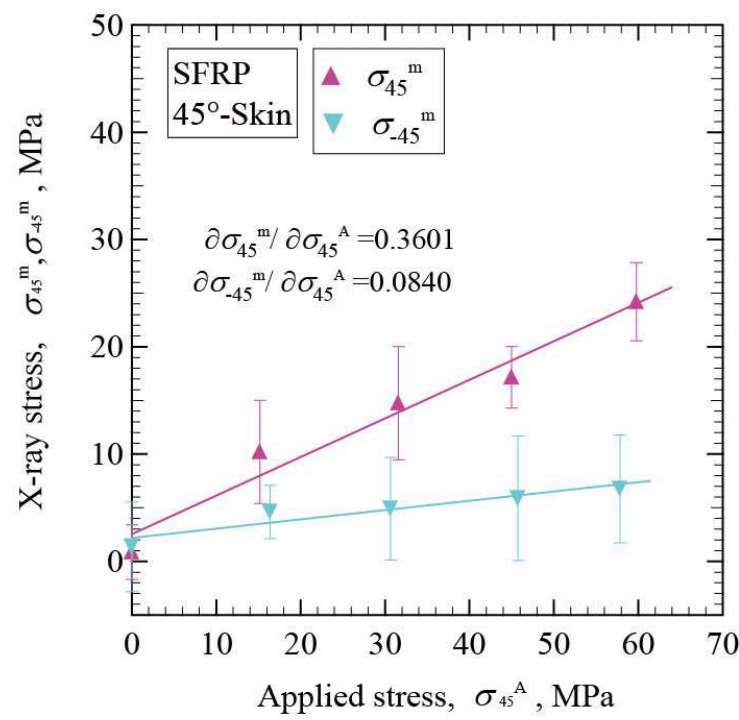

(c) $45^{\circ}$ speciemn

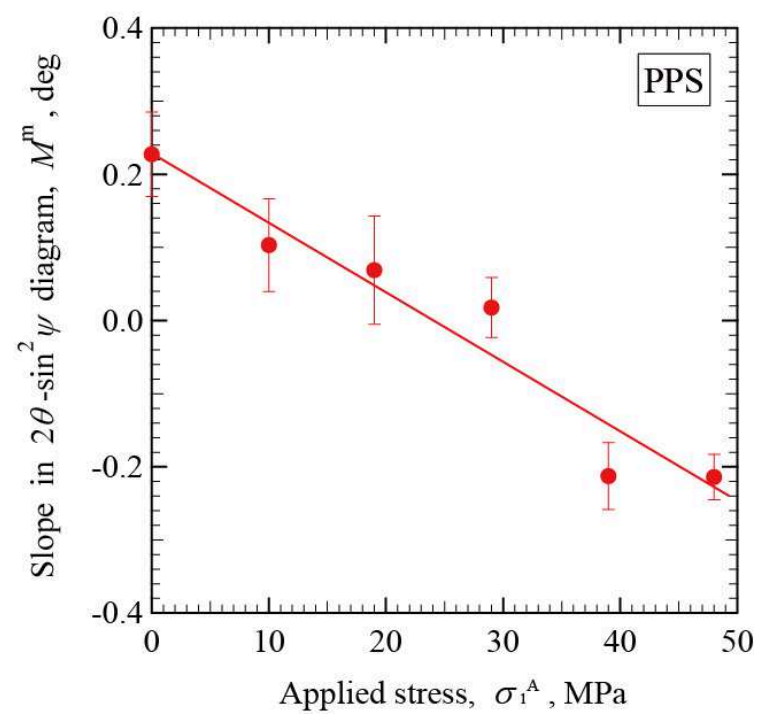

Fig. 3 Changes of slope with applied strain for PPS.

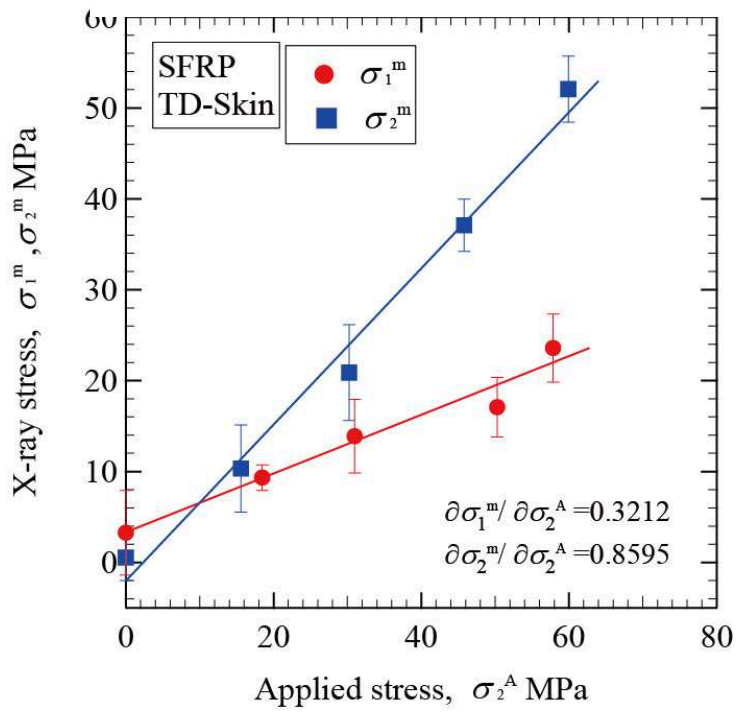

(b) TD speciemn

Fig. 4 Matrix stress as a function of applied stress for three types of SFRP. 
Proposed Method of Residual Stress Measurement of SFRP. Residual stresses on the surface of SFRP components are expected to be biaxial plane stress state. Once the fiber direction is known at the measurement points, the macrostress, $\sigma_{1}^{A}$ and $\sigma_{2}^{A}$, parallel and perpendicular to fibers can be determined from the measurements of the matrix stresses, $\sigma_{1}^{m}$ and $\sigma_{2}^{m}$, in two directions by

$$
\begin{aligned}
& \sigma_{1}^{A}=\frac{\alpha_{22} \sigma_{1}^{m}-\alpha_{12} \sigma_{2}^{m}}{\alpha_{11} \alpha_{22}-\alpha_{12} \alpha_{21}}, \\
& \sigma_{2}^{A}=\frac{\alpha_{11} \sigma_{2}^{m}-\alpha_{21} \sigma_{1}^{m}}{\alpha_{11} \alpha_{22}-\alpha_{12} \alpha_{21}} .
\end{aligned}
$$

The shear stress, $\tau_{12}^{A}$, is determined from the matrix stresses, $\sigma_{+45}^{m}, \sigma_{-45}^{m}$, measured in two directions $\pm 45^{\circ}$ to the fiber direction by using the following equation:

$$
\tau_{12}^{A}=\left(\sigma_{+45}^{m}-\sigma_{-45}^{m}\right) / 2 \alpha_{55} .
$$

The application of the present method to SFRP components in ground vehicles will be conducted in the future.

\section{Summary}

A new method was proposed to evaluate the macrostress in SFRP from the measurement of the matrix stress. The matrix stress in crystalline thermoplastics PPS was determined with the $\sin ^{2} \psi$ method.

(1) The matrix stresses in the fiber direction and perpendicular to the fiber direction, and the shear stress in SFRP are expressed as the proportional functions of the applied (macro-) stresses and the proportional constants were determined experimentally.

(2) Once the fiber direction is known at the measurement position of the stress in SFRP, the residual stress in the skin layer of SFRP can be evaluated from the matrix stress determined by X-rays in four directions: the directions parallel, perpendicular and $\pm 45^{\circ}$ to the fibers.

\section{Appendix - Micromechanics of SFRP}

Fig. 5 shows a model SFRP where fibers are represented by prolate spheroids aligned along the molding direction. The stress in matrix and fiber phases can be calculated as a function of the applied stress based on micromechanics of Eshelby's ellipsoidal inclusions [8] combined with the Mori-Tanaka mean stress theory [9]. The matrix is assumed to have isotropic elastic constant $C_{0}$, and the fiber has orthogonally anisotropic elastic constant $C_{f}$. When the stress, $\sigma_{0}\left(=\sigma^{\mathrm{A}}\right)$ is applied to SFRP, the strain, $e_{0}$, of the matrix without fibers is related to the applied stress. In SFRP, the matrix stress is perturbed by fibers and the additional stress, $\tilde{\sigma}$, is introduced in the matrix. The strain, $\tilde{e}$, associated to the stress $\tilde{\sigma}$. The matrix stress, $\langle\sigma\rangle^{m}$, is expressed by

$$
\langle\sigma\rangle^{m}=\sigma_{0}+\tilde{\sigma}=C_{0} \cdot\left(e_{0}+\tilde{e}\right)
$$

Since the s additional strain, $e$, is introduced in fiber phase due to the mismatch of elastic constant, the mean stress in the fiber phase is

$$
\langle\sigma\rangle^{f}=\sigma_{0}+\tilde{\sigma}=C_{f} \cdot\left(e_{0}+\tilde{e}+e\right) \text {. }
$$

The fiber stress can be expressed by equivalent inclusions with the eigen strain $e^{*}$, as follows [8]:

$$
C_{f} \cdot\left(e_{0}+\tilde{e}+e\right)=C_{0} \cdot\left(e_{0}+\tilde{e}+e-e^{*}\right) .
$$

The additional strain, $e$, is related to eigen strain $e^{*}$ through

$$
e=S \cdot e^{*} \text {. }
$$

where $S$ is Eshelby's tensor. According to Mori-Tanaka mean stress theory, the volumetric integral of the additional stress, $\tilde{\sigma}$, is zero. From this condition, the strain, $\tilde{e}$, is determined as follows:

$$
\tilde{e}=-f\left(e-e^{*}\right) \text {. }
$$




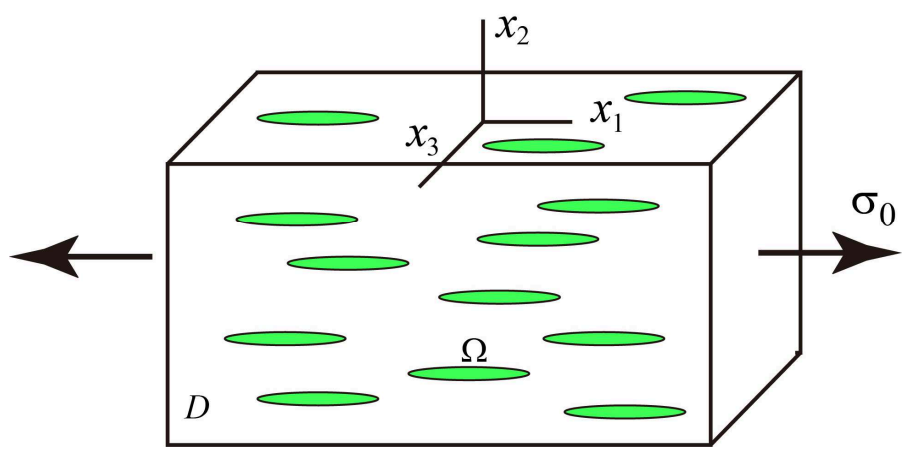

Fig. 5 Calculation model of elastic deformation of SFRP.

(The fibers are aligned along the molding direction, $x_{1}$ axis.)

By substituting Eqs. (15), (16) into Eq. (14), the eigen strain is determined as

$$
e^{*}=-\left[\left(C_{f}-C_{0}\right) \cdot\{(1-f) S+f I\}+C_{0}\right]^{-1} \cdot\left(C_{f}-C_{0}\right) \cdot e_{0} \equiv A \cdot e_{0}
$$

The matrix stress is given from Eqs. (12), (16), (17) as follows:

$$
\langle\sigma\rangle^{m}=C_{0} \cdot\left(e_{0}+\tilde{e}\right)=C_{0} \cdot[I-f(S-I) \cdot A] \cdot e_{0}=C_{0} \cdot[I-f(S-I) \cdot A] \cdot C_{0}^{-1} \cdot \sigma_{0} \equiv F \cdot \sigma_{0} .
$$

The above equation gives the relation between the matrix stress and the applied stress. A matrix representation of the above relation is

$$
\left[\begin{array}{c}
\sigma_{1}^{m} \\
\sigma_{2}^{m} \\
\sigma_{3}^{m} \\
\tau_{23}^{m} \\
\tau_{31}^{m} \\
\tau_{12}^{m}
\end{array}\right]=\left[\begin{array}{cccccc}
\alpha_{11} & \alpha_{12} & \alpha_{12} & 0 & 0 & 0 \\
\alpha_{21} & \alpha_{22} & \alpha_{23} & 0 & 0 & 0 \\
\alpha_{21} & \alpha_{23} & \alpha_{22} & 0 & 0 & 0 \\
0 & 0 & 0 & \alpha_{44} & 0 & 0 \\
0 & 0 & 0 & 0 & \alpha_{55} & 0 \\
0 & 0 & 0 & 0 & 0 & \alpha_{55}
\end{array}\right] \cdot\left[\begin{array}{c}
\sigma_{1}^{0} \\
\sigma_{2}^{0} \\
\sigma_{3}^{0} \\
\tau_{23}^{0} \\
\tau_{31}^{0} \\
\tau_{12}^{0}
\end{array}\right] .
$$

For the case of plane stress, the matrix stress are given expressed as

$$
\begin{aligned}
& \sigma_{1}^{m}=\alpha_{11} \sigma_{1}^{0}+\alpha_{12} \sigma_{2}^{0}, \\
& \sigma_{2}^{m}=\alpha_{21} \sigma_{1}^{0}+\alpha_{22} \sigma_{2}^{0}, \\
& \tau_{12}^{m}=\alpha_{55} \tau_{12}^{0} .
\end{aligned}
$$

In the main context, the applied stress is expressed as $\sigma^{\mathrm{A}}$ in stead of $\sigma^{0}$. It is interesting to note that the applied normal stresses perpendicular and parallel to fibers induce only normal stresses perpendicular and parallel to the fibers, but not the shear stress, in the matrix.

\section{References}

[1] J. Karger-Kocsis, Fracture of short-fiber reinforced thermoplastics, in: K. L. Reifsnider (Ed.), Application of Fracture Mechanics to Composite Materials, L., Elesevier Science Pub., Amsterdam, (1989) 189-247.

[2] J. F. Mandell, Fatigue behavior of short fiber composites materials, in: K. L. Reifsnider (Ed.), Fatigue of Composites, Elesevier Science Pub., Amsterdam, (1990) 231-337.

[3] J. Karger-Kocsis and K. Friedlich, Microstructural details and the effect of testing conditions on the fracture toughness on injection-molded poly(phenylene-sulphide) composites, Journal of Materials Science, Vol. 22 (1987) 947-961. 
[4] K. Tanaka, K. Suzuki and Y. Akiniwa, Evaluation of Residual Sresses by X-Ray Diffraction, Yokendo, Tokyo (2006).

[5] K. Tanaka, S. Tokoro, N. Egami and Y. Akiniwa, X-ray residual stress measurement of short-fiber reinforced plastics, Proceeding of the $47^{\text {th }}$ Symposium of X-Ray Studies of Mechanical Behavior of Materials, The Society of Materials Science, Japan, (2013) 119-124.

[6] B. J. Tabor, E. P. Magre and J. Boon, The crystal structure of poly-p-phenylene sulphide, European Polymer Journal, Vol. 7 (1971) 1127-1133.

[7] J. S. Chung, J. Bodzich and P. Chee, Effects of thermal history on crystal structure of poly(phenylene sulphide), Journal of Materials Science, Vol. 27 (1992) 5609-5619.

[8] J. D. Eshelby, The determination of the elastic field of an ellipsoidal inclusion, and related problems, Proceedings of Royal Society, London, Vol. A241 (1957) 376-396.

[9] T. Mori and K.Tanaka, Average stress in matrix and average energy of materials with misfitting inclusions, Acta Metallugica, Vol. 21 (1973) 571-574. 